\title{
Correction to: Heterogenous chemosensitivity of a panel of organoid lines derived from small cell neuroendocrine carcinoma of the uterine cervix
}

\author{
Mie Tanaka ${ }^{1,5} \cdot$ Jumpei Kondo $^{1,2} \cdot \mathrm{Kensuke} \mathrm{Kaneko}^{4} \cdot \mathrm{Hiroko} \mathrm{Endo}^{2,6} \cdot \mathrm{Kunishige} \mathrm{Onuma}^{1} \cdot$ Roberto Coppo $^{1}$. \\ Masamune Masuda $^{1,5} \cdot$ Shoji Kamiura $^{3} \cdot$ Kiyoshi Yoshino $^{5,7} \cdot$ Yutaka Ueda $^{5} \cdot$ Hideaki Kakeya $^{4} \cdot$ Tadashi Kimura $^{5}$. \\ Masahiro Inoue ${ }^{1,2}$ (1)
}

Published online: 8 April 2021

(c) Japan Human Cell Society 2021

\section{Correction to: Human Cell}

https://doi.org/10.1007/s13577-021-00511-5

In the original publication of the article, the legend of the Y-axis in figure $5 \mathrm{~d}$ and figure S2 should be "Absorbance at $405 \mathrm{~nm}$ " instead of "CES activity (U/mg protein)". In the Fig. 5 legend, "d" is mislabelled as "b".

The correct Fig. 5 and Fig. s2 are given in this erratum.

The original article can be found online at https://doi.org/10.1007/ s13577-021-00511-5.

Masahiro Inoue

masa_inoue@kuhp.kyoto-u.ac.jp

1 Department of Clinical Bio-Resource Research and Development, Graduate School of Medicine Kyoto University, 46-29 Shimoadachi-cho, Sakyou-ku, Kyoto 606-8304, Japan

2 Department of Biochemistry, Osaka International Cancer Institute, Osaka, Japan

3 Department of Gynecology, Osaka International Cancer Institute, Osaka, Japan

4 Department of System Chemotherapy and Molecular Sciences, Division of Bioinformatics and Chemical Genomics, Graduate School of Pharmaceutical Sciences, Kyoto University, Kyoto, Japan

5 Department of Obstetrics and Gynecology, Osaka University Graduate School of Medicine, Suita, Japan

6 Present Address: Carna Biosciences Inc, Hyogo, Japan

7 Present Address: Department of Obstetrics and Gynecology, University of Occupational and Environmental Health, Fukuoka, Japan 
a



b

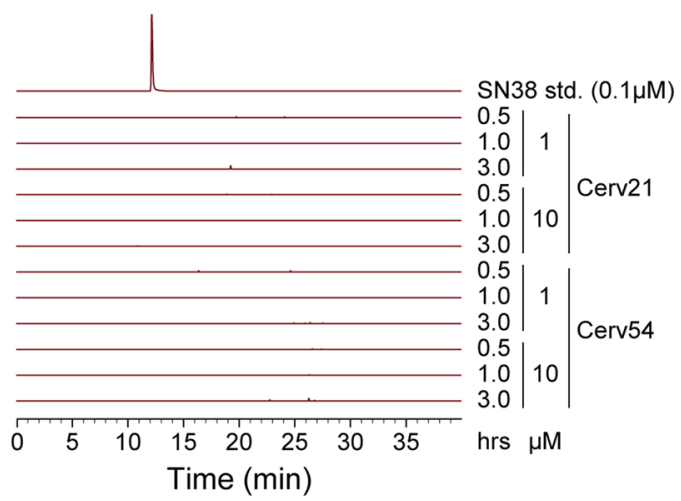

Irinotecan $10 \mu \mathrm{M}$

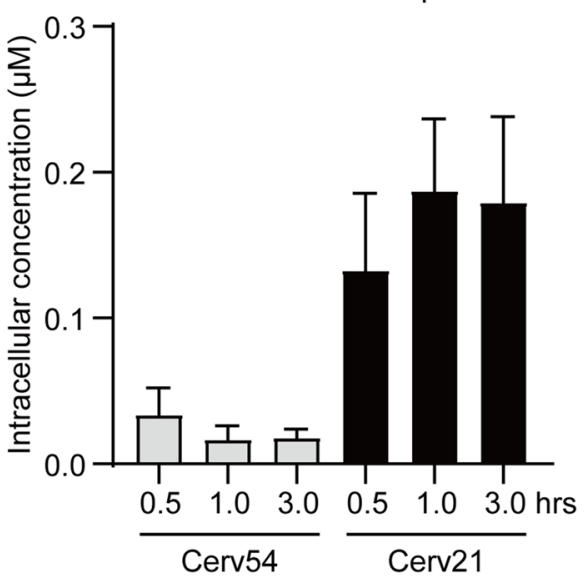

C



菂

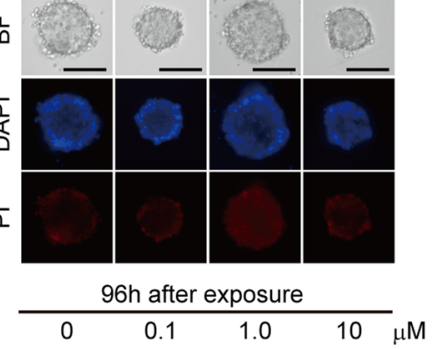

㭊



d

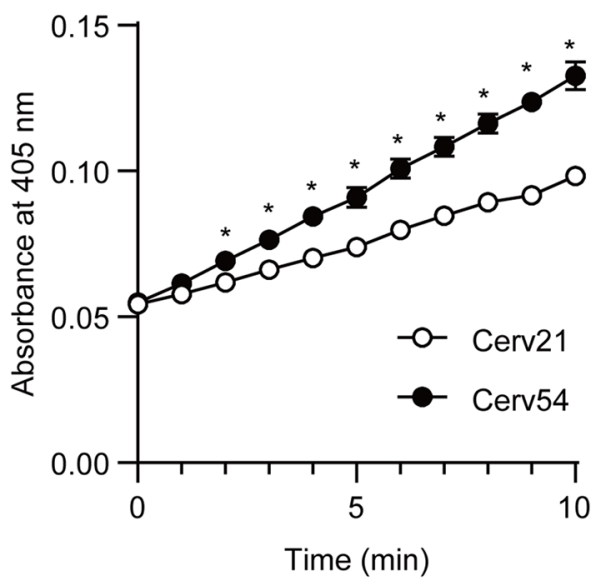

e

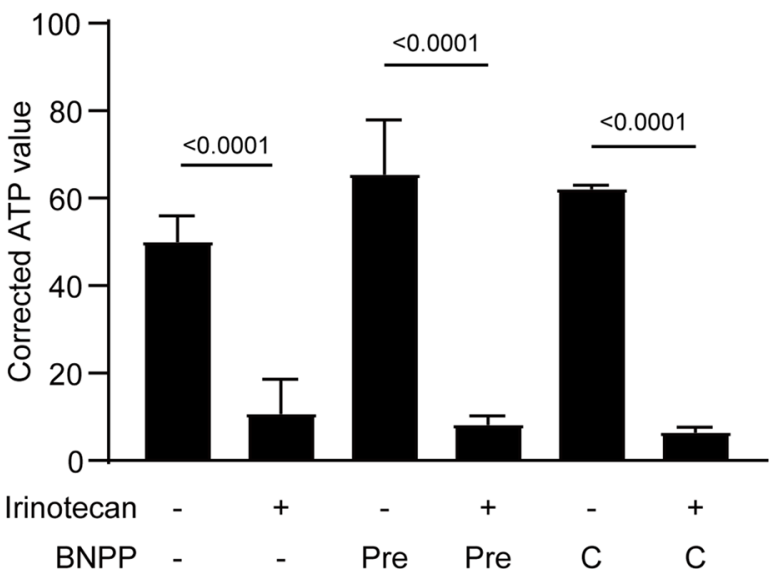




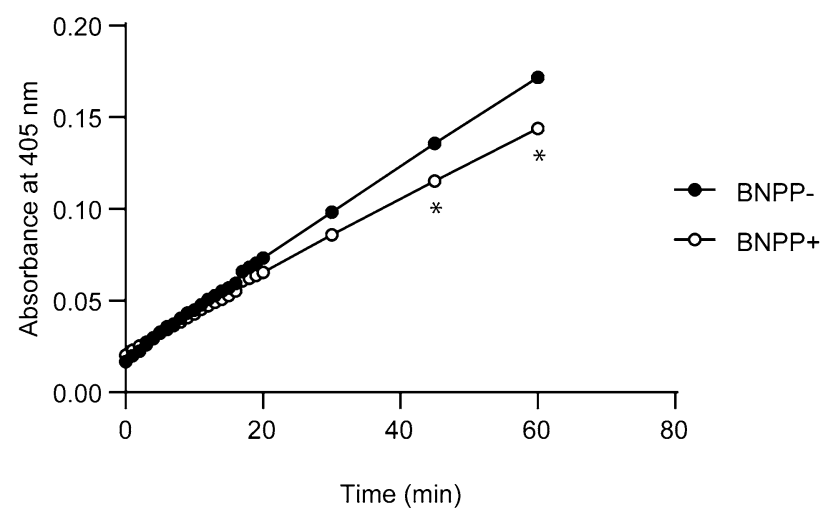

Publisher's Note Springer Nature remains neutral with regard to jurisdictional claims in published maps and institutional affiliations. 